Gutiérrez-de-Rozas, B. y Carpintero Molina, E. (2021). Análisis de la evaluación de programas de intervención èn motivación en Educación Secundaria. Revista de Investigación Educativa, 39(2), 503-525.

DOI: http://dx.doi.org/10.6018/rie.442341

\title{
Análisis de la evaluación de programas de educación emocional y motivación en Educación Secundaria
}

\section{Analysis of the evaluation of emotional education and motivation programs in Secondary Education}

\author{
Belén Gutiérrez-de-Rozas' y Elvira Carpintero Molina \\ *Departamento de Métodos de Investigación y Diagnóstico en Educación II, Facultad de Educación, Universidad \\ Nacional de Educación a Distancia (UNED) (España) \\ **Departamento de Investigación y Psicología en Educación, Facultad de Educación, Universidad Complutense de \\ Madrid (España)
}

\begin{abstract}
Resumen
La motivación y las emociones son aspectos clave en el ámbito escolar debido a su clara relación con el rendimiento académico, y los programas de intervención se proponen como un recurso adecuado para contribuir a su mejora y ajuste. Sin embargo, seleccionar el programa adecuado y con garantías de calidad no siempre es una tarea sencilla. Una manera de obtener cierta garantía es partir de aquellos programas de intervención que no solo han sido evaluados, sino de los que además se dispone de evidencias de que dicha evaluación se ha realizado de forma correcta.

El presente trabajo tiene como objetivo analizar los procesos y resultados de evaluación de diferentes programas de educación emocional y de motivación dirigidos a estudiantes de Secundaria y/o adolescentes a partir de los resultados de sus evaluaciones. Para ello, se realiza una aproximación teórica a la evaluación y metaevaluación de programas y se lleva a cabo una revisión de la literatura académica de la que se seleccionan y comparan evaluaciones de programas de educación emocional y motivación. Finalmente, se realiza una metaevaluación mediante la aplicación de estándares.
\end{abstract}

Correspondencia: Belén Gutiérrez-de-Rozas, bgutierrezderozas@edu.uned.es, calle Juan del Rosal, 14, 28040 Madrid, España. 
Los resultados muestran que las evaluaciones se realizan, generalmente, de forma interna y sumativa, centrándose en los resultados de aplicación y no tanto en el proceso.

Palabras clave: evaluación; metaevaluación; intervención; motivación; emoción; enseñanza secundaria

\section{Abstract}

Motivation and emotions are key aspects in education due to their influence on academic performance, and intervention programs are a useful resource to contribute to their improvement. However, selecting the right programme with quality guarantees is not always a simple task. One way to have a certain guarantee is by choosing those programs that have not only been evaluated, but for which there is also evidence that the evaluation has been performed correctly

The present work aims to analyse the evaluation processes and results of different motivation and emotional education programs addressed to Secondary students and/or adolescents from the results of their evaluation results. In order to achieve this goal, a theoretical approach to program evaluation and meta-evaluation is made, as well as a review of the academic literature from which evaluations of emotional education and motivation programs are selected and compared. Finally, a meta-evaluation is performed through the application of standards.

The results show that evaluations are generally conducted internally and in a summative manner, focusing on the results of implementation rather than on the process.

Keywords: evaluation; metaevaluation; intervention; learning motivation; emotion; secondary education

\section{Introducción}

Las emociones y la motivación tienen papeles esenciales a la hora de determinar el rendimiento académico de los estudiantes. Las emociones implican cambios en los sentimientos, cogniciones, tendencias de acción, comportamientos y aspectos fisiológicos a raíz de un efecto precedente (Lange et al., 2020) y poseen una influencia clave en el rendimiento, ya que los procesos educativos siempre están acompañados de estados emocionales (Pulido Acosta y Herrera Clavero, 2017) que regulan las acciones de los estudiantes. Por su parte, la motivación es el impulso para actuar que sucede a raíz de la experimentación de dichas emociones (Anaya, 2014; Mirete Ruiz et al., 2015) y ha centrado el interés de la comunidad científica no solo desde hace décadas (Järvela, 2001; López, 2006; Rinaudo et al., 2003) sino, también, en los últimos tiempos (Barati, Joo y Aria, 2018; De Caso, 2014). Este constructo influye tanto en los procesos cognitivos y en las estrategias de aprendizaje de los estudiantes como en sus actitudes futuras hacia el aprendizaje (García, 2008).

Estos aspectos, si bien deben ser considerados en todas las etapas educativas, cobran especial relevancia en Educación Secundaria puesto que, en ella, los menores se encuentran experimentando cambios emocionales y motivacionales propios de la adolescencia (Burunat, 2004), la cual abarca desde los 10 años hasta los 19 (OMS, 2020) o según Sawyer et al. (2016), hasta los 24. Por tanto, resulta clave promover medidas para su mejora. 
Una de las herramientas que permiten mejorar estos aspectos a través de acciones coherentes y organizadas son los programas de intervención, definidos como unidades de acción que contribuyen a alcanzar de manera sistemática objetivos previamente planteados (Pérez Juste, 2000; 2006) en función de las necesidades detectadas (Martínez Mediano, 2017). Para alcanzar dichos objetivos, es imprescindible que los programas estén diseñados e implementados de manera correcta, garantizando que con su aplicación se logren los resultados esperados. Por ello, uno de los elementos esenciales que debe considerarse a la hora de seleccionar un programa de intervención es si cuenta con un proceso de evaluación riguroso que incluya la recogida de información válida y fiable y que permita determinar la calidad y los logros para tomar decisiones de mejora (Pérez Juste, 2000) y la magnitud de los cambios producidos (Frye y Hammer, 2012).

La necesidad de conocer el impacto de los programas, así como de rendir cuentas a la sociedad, ha contribuido al aumento de la importancia de la práctica de la evaluación (Méndez Pineda, 2018). Pese a ello, tal y como fue detectado por Hernández y Álvarez Rojo (1998) y corroborado posteriormente por Expósito et al. (2004), el desequilibrio entre la cantidad existente de programas de intervención evaluados y no evaluados es manifiesto. En esta línea, Pérez-González (2008) explica que la evaluación de programas en el ámbito educativo es limitada y contrasta con otros ámbitos como el de la agricultura o la medicina. Para ello, pone como ejemplo la revisión llevada a cabo por Vallés y Vallés (2003), en la que únicamente uno de los catorce programas de educación emocional contemplados presentó estudios de validación. Además, SánchezCalleja et al. (2016) realizan una revisión de programas de educación emocional en la que observan que estos no siempre cuentan con evaluaciones y, en caso de hacerlo, no poseen el nivel de profundidad adecuado.

Con el objetivo de indagar en qué medida los programas de educación emocional y de mejora de la motivación ofrecen resultados de evaluación, y cuáles son las características de estos datos, se presenta este artículo.

\section{Evaluación de programas}

La literatura académica muestra diversas perspectivas desde las que abordar la evaluación de programas. Por su relevancia, cabe destacar aquellos modelos que tradicionalmente han servido de base a la teoría evaluativa, como el modelo de cuatro niveles de Kirkpatrick (1959), el modelo Contexto, Input, Proceso y Producto (CIPP) de Stufflebeam $(1971,1987)$ o el modelo de Stake (1975), centrado en los antecedentes, el proceso, las normas, los juicios y los resultados. Según Pérez Juste (1995, 2000, 2006), todo programa educativo ha de evaluarse en tres momentos, que constituyen la evaluación inicial, procesual o continua, y final o sumativa. Para ello, Pérez Juste (2006) desarrolla una pauta de evaluación que integra 74 indicadores agrupados en cuatro categorías relacionadas con estos tres momentos y la institucionalización de la evaluación.

Por su parte, Álvarez Rojo et al. (2002) determinan los supuestos metodológicos de la evaluación de programas, clasificando las evaluaciones en función del agente, la función de la evaluación, la perspectiva temporal y los elementos del programa que se estén evaluando (Tabla 1). 
Tabla 1

Tipos de evaluación de programas.

\begin{tabular}{|c|c|c|}
\hline Criterio & Tipos & Características \\
\hline \multirow[t]{2}{*}{ Evaluador } & Evaluación interna & $\begin{array}{l}\text { Realizada por el mismo sujeto que } \\
\text { diseña y/o aplica el programa }\end{array}$ \\
\hline & Evaluación externa & $\begin{array}{l}\text { Realizada por sujetos distintos de } \\
\text { quien diseña y/o aplica el programa }\end{array}$ \\
\hline \multirow[t]{2}{*}{$\begin{array}{l}\text { Función de la } \\
\text { evaluación }\end{array}$} & Evaluación formativa & $\begin{array}{l}\text { Dirigida al desarrollo y mejora del } \\
\text { programa }\end{array}$ \\
\hline & Evaluación sumativa & $\begin{array}{l}\text { Dirigida al control y valoración de } \\
\text { resultados }\end{array}$ \\
\hline \multirow[t]{2}{*}{$\begin{array}{l}\text { Perspectiva } \\
\text { temporal }\end{array}$} & Evaluación ex ante & $\begin{array}{l}\text { Realizada antes de la aplicación } \\
\text { para considerar la pertinencia y } \\
\text { probabilidad de éxito }\end{array}$ \\
\hline & Evaluación ex post & $\begin{array}{l}\text { Realizada cuando el programa ha } \\
\text { concluido }\end{array}$ \\
\hline \multirow[t]{3}{*}{$\begin{array}{l}\text { Elementos del } \\
\text { programa }\end{array}$} & $\begin{array}{l}\text { Evaluación de las necesidades o } \\
\text { del diseño }\end{array}$ & Realizada en la etapa de planificacón \\
\hline & $\begin{array}{l}\text { Evaluación de la implementación, } \\
\text { la cobertura y el seguimiento }\end{array}$ & Realizada la etapa de aplicación \\
\hline & $\begin{array}{l}\text { Evaluación de los resultados y el } \\
\text { impacto }\end{array}$ & Realizada en la etapa de finalización \\
\hline
\end{tabular}

Fuente: Álvarez Rojo et al. (2002).

\section{Metaevaluación de programas}

El concepto de metaevaluación, acuñado por Scriven (1967), hace referencia a una investigación que, de manera sistemática, busca realizar un juicio de valor sobre la calidad y los méritos de una evaluación, teniendo como objetivo asegurar la calidad de los servicios evaluativos, evitar las prácticas ilegales e indicar el camino para perfeccionar la profesión evaluadora, así como favorecer su comprensión (Stufflebeam, 1987).

Con el fin de que la metaevaluación de programas se lleve a cabo con éxito, se han desarrollado una serie de estándares de evaluación que sirven como marco de trabajo, así como de guía sobre aquellos aspectos que permiten determinar su calidad. A la hora de realizar una metaevaluación, es necesario considerar que no todos los estándares han de ser aplicados en cada uno de los procesos de evaluación. Por el contrario, los profesionales encargados de su realización deben seleccionar y priorizar aquellos que se ajusten a las características de su evaluación (Samson y Solabarrieta, 2018) o que sean aplicables en el contexto en el que esta se desarrolla (Martínez Mediano, 2017). 
Cabe resaltar, debido a su actualidad, los Estándares del Joint Committee on Standards for Educational Evaluation (Yarbrough et al., 2011), compuestos por 30 ítems clasificados en cinco grupos: utilidad, viabilidad, adecuación, exactitud y responsabilidad; y la MetaEvaluation Checklist (Scriven 2013), que divide los criterios de metaevaluación en cuatro grupos: preliminares, fundamentos, subevaluaciones y conclusiones e implicaciones. El grupo de criterios preliminares se relaciona con la necesidad de explicitar los pasos y la metodología que sigue un programa, así como la justificación de la evaluación; se compone de los estándares A1 (Resumen ejecutivo), A2 (Clarificaciones) y A3 (Diseño y métodos). El grupo fundamentos está formado por los estándares B1 (Antecedentes y contexto), B2 (Descripciones y definiciones), B3 (Consumidores), B4 (Recursos) y B5 (Valor), que se refieren a la especificación de los elementos del programa, tales como su contexto y su naturaleza. El tercer grupo de subevaluaciones se vincula con el momento de observación de las evidencias. Se contempla la evaluación tanto durante el proceso de aplicación como en relación con el impacto y la posibilidad de establecer comparaciones o aplicar el programa a otros contextos. Está formado por los estándares C1 (Procesos), C2 (Resultados), C3 (Costes), C4 (Comparaciones) y C5 (Generalizaciones).

El último grupo de criterios, conclusiones e implicaciones, se relaciona con las consideraciones previas a la elaboración del informe de resultados y contempla también la realización de metaevaluaciones durante el proceso; se compone de los estándares D1 (Síntesis), D2 (Recomendaciones, explicaciones, predicciones y rediseños), D3 (Responsabilidad y justificación), D4 (Informe y apoyo) y D5 (Metaevaluación).

\section{Método}

\section{Objetivos}

El presente trabajo tiene como objetivo analizar los procesos y resultados de evaluación de diferentes programas de educación emocional y de mejora de la motivación dirigidos a estudiantes de Secundaria y/o adolescentes.

\section{Muestra}

La muestra objeto de estudio se compone de un total de 19 documentos que describen la evaluación de programas de intervención en motivación y en educación emocional. La selección de la muestra partió de una búsqueda inicial en las bases de datos ERIC y Google Académico. Como criterio de inclusión, se seleccionaron aquellos documentos publicados con posterioridad al año 2000 en formato artículo, informe, tesis doctoral o actas de congresos, excluyendo los documentos en formato libro o manual. Las palabras clave utilizadas fueron: evaluación, programa, secundaria, motivación y emoción, tanto en castellano como en inglés.

Tras el proceso de búsqueda inicial, se consideraron 374 resultados (fase 1), de los cuales se seleccionaron únicamente aquellos que cumplían los criterios relacionados tanto con las características de los programas como con su evaluación (fase 2):

- Destinatarios: alumnado de Educación Secundaria y/o adolescentes (10-19 años).

- Contexto: instituciones y centros educativos de educación formal y no formal. 
- Elementos considerados en la evaluación: aspectos emocionales y motivacionales.

Una vez aplicados estos criterios, el número de publicaciones se redujo a 153 documentos. Tras ello, se eliminaron aquellos documentos que abordaban intervenciones sobre poblaciones específicas (alumnado con discapacidad, estudiantes con altas capacidades...), excluyendo 56 artículos y configurando un total de 97 documentos (fase 3).

Finalmente, se realizó un último proceso en el que se seleccionaron aquellos artículos en los que se indicaba de forma identificable el nombre del programa evaluado. Además, se descartaron aquellos que mostraban evaluaciones de programas aplicados antes del año 2000 (fase 4). Estos criterios eliminaron una cifra elevada de artículos (78), estableciendo una muestra final de 19 documentos (ver Figura 1).

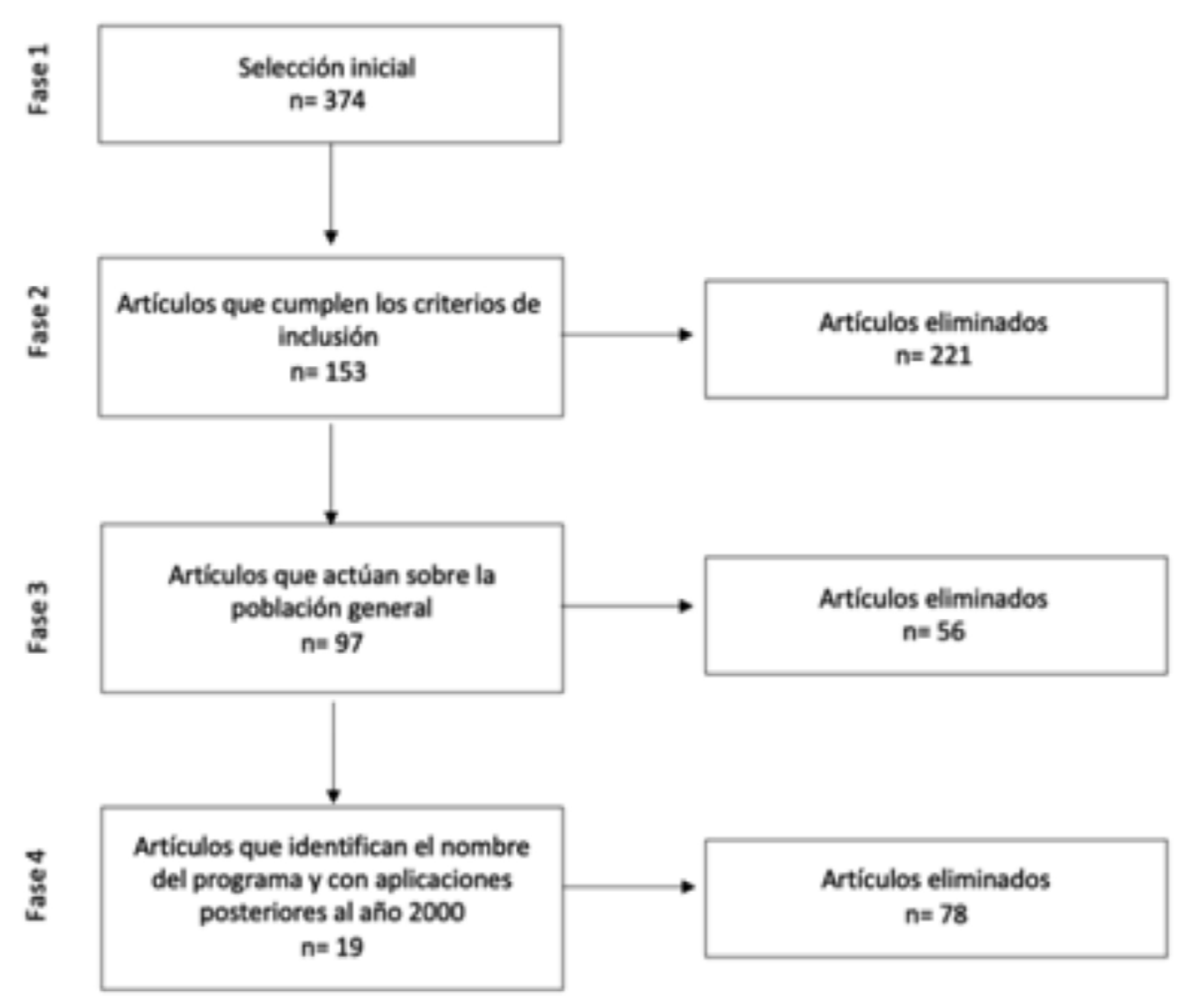

Figura 1. Proceso de identificación y selección

\section{Procedimiento de recogida y análisis de datos}

Una vez identificados y seleccionados los documentos, se procedió a analizarlos atendiendo a los siguientes criterios: año y lugar de realización de la evaluación, 
diseño, muestra, instrumentos, características del evaluador, función de la evaluación, perspectiva temporal y elementos evaluados (ver tabla 2).

Tabla 2.

Criterios de clasificación y categorías de respuesta asociadas.

\begin{tabular}{cc}
\hline Criterio & Categorías \\
\hline Año de publicación de la evaluación & $2000-2018$ \\
\hline País de evaluación & Respuesta abierta \\
\hline Diseño y metodología & Cualitativa, cuantitativa, mixta \\
\hline Muestra & Respuesta abierta \\
\hline Instrumentos & Respuesta abierta \\
\hline Evaluador & Interno, externo \\
\hline Función & Formativa, sumativa \\
\hline Perspectiva temporal & Ex post, ex ante \\
\hline Elementos evaluados & Necesidades, implementación, resultados \\
\hline
\end{tabular}

En el Apéndice se muestran los resultados del análisis de cada una de las dimensiones para las evaluaciones contempladas ${ }^{1}$.

De forma complementaria, se llevó a cabo una metaevaluación aplicando los estándares de la Meta-Evaluation Checklist (Scriven, 2013). Se trata, en todo caso, de una metaevaluación externa desarrollada tras la finalización del proceso de aplicación del programa y del proceso de evaluación a partir de la información disponible en los documentos, por lo que se seleccionan, únicamente, los estándares aplicables al presente trabajo (A3, B1, B2, B3, C1, C2, D1, D3 y D4) y se omiten aquellos que deben ser aplicados durante el proceso de evaluación o para los que no se dispone de información suficiente a través de las publicaciones seleccionadas (A1, A2, B4, B5, C3, C4, C5, D2 y D5).

\section{Resultados}

\section{Análisis de la evaluación de los programas}

A continuación, se presentan los resultados del estudio atendiendo a los criterios inicialmente señalados.

Como consecuencia del criterio de actualidad aplicado, los documentos aparecen principalmente a partir del año 2012, y, más concretamente, se incrementa el número de publicaciones a partir de 2015 (Tabla 3).

1 Debe considerarse que los datos han sido obtenidos a partir del análisis de los documentos seleccionados, por lo que es posible que existan datos complementarios que no se han contemplado en este estudio. 
Tabla 3.

Distribución de porcentajes del año de publicación de los artículos de evaluación de los programas².

\begin{tabular}{c|r|r|r|r|r|r|r|r}
\hline Año & 2008 & 2011 & 2012 & 2013 & 2015 & 2016 & 2017 & 2018 \\
\hline$\%$ & 5,3 & 5,3 & 10,5 & 5,3 & 31,5 & 15,8 & 15,8 & 15,8 \\
\hline
\end{tabular}

En lo que respecta al país de aplicación y realización de las evaluaciones, el 47,3\% de los documentos identificados pertenecen a España y el 26,3\% a Estados Unidos, mientras que el 5,3\% de los programas se han evaluado en Portugal, Reino Unido, Macedonia, Letonia y Dinamarca, respectivamente.

Atendiendo al diseño de evaluación de resultados, la mayoría de las evaluaciones plantean un diseño pre test-post test, comparándose las medidas tomadas antes de la aplicación del programa con aquellas resultantes de dicha aplicación (73,7\%). En el $57,9 \%$ se concreta el diseño de dos grupos (control y experimental no equivalente en el $47,4 \%$ de los casos; control y experimental equivalente en el 10,5\%), mientras que el $26,3 \%$ no cuenta con grupo de control. En la mayor parte de las evaluaciones se ha seguido una metodología cuantitativa (47,5\%), mientras que el 36,8\% han empleado una metodología mixta y tan solo el 5,3\% una metodología cualitativa.

Entre los instrumentos de evaluación se han utilizado pruebas estandarizadas (26,3\%) y no estandarizadas (21\%), destacando la combinación de ambos tipos de instrumentos $(42,1 \%)$, si bien en el 10,5\% no se explicita el tipo de instrumento.

Con respecto al evaluador, el 52,6\% de los programas cuenta con un evaluador interno (generalmente, el diseñador y/o aplicador del programa), en el 21\% la evaluación es externa y en el $26,4 \%$ restante la evaluación se ha llevado a cabo por evaluadores externos e internos de manera conjunta. Con respecto a la función de la evaluación, el 78,9\% de los programas tiene una función sumativa, evaluándose, por tanto, sus resultados. Únicamente en el 5,3\% la evaluación es formativa, mientras que en el 15,8\% es tanto formativa como sumativa.

En lo relativo a la perspectiva temporal, esto es, el momento en el que se ha llevado a cabo la evaluación, en el 78,9\% de los programas la evaluación es realizada ex post o tras la aplicación del programa; en el 5,3\% de los casos se lleva a cabo ex ante o previamente a la aplicación, y solo en el 15,8\% se realiza en ambos momentos.

Por último, atendiendo a los elementos evaluados y vinculado con la perspectiva temporal, en el 57,9\% de los programas se busca conocer los resultados de la aplicación, mientras que solo en el 5,3\% se ha evaluado el diseño y en otro 5,3\%, la implementación, si bien cabe destacar que en el $26,3 \%$ se ha evaluado tanto la implementación como los resultados y en el 5,3\%, los tres elementos.

La Tabla 4 ilustra los resultados en función del tipo de evaluador, la función, la perspectiva temporal y los elementos evaluados.

2 Se han representado, únicamente, aquellos años en los que se han encontrado evaluaciones de programas. 
Tabla 4.

Resultados del tipo de evaluador, función de la evaluación, perspectiva temporal y elementos evaluados.

\begin{tabular}{llc}
\hline Elemento & Tipología & \% \\
\hline Tipo de evaluador & Interno & 52,6 \\
& Externo & 21 \\
& Interno y externo & 26,4 \\
\hline \multirow{2}{*}{ Función de la evaluación } & Formativa & 5,3 \\
& Sumativa & 78,9 \\
& Formativa y sumativa & 15,8 \\
\hline Perspectiva temporal & Ex ante & 5,3 \\
& Ex post & 78,9 \\
& Ex ante y ex post & 15,8 \\
\hline Elementos evaluados & Diseño & 5,3 \\
& Implementación & 5,3 \\
& Resultados & 57,9 \\
& Implementación y resultados & 26,3 \\
& Diseño, implementación y resultados & 5,3 \\
\hline
\end{tabular}

\section{Metaevaluación}

Dada la importancia que posee la metaevaluación a la hora de garantizar la calidad y la honradez de las evaluaciones, se ha procedido a la aplicación de los estándares de la Meta-Evaluation Checklist (Scriven, 2013) en cada una de las 19 evaluaciones de programas.

En la Tabla 5 se recogen los estándares aplicados y el porcentaje de programas que cumplen cada uno de ellos.

Tabla 5.

Estándares aplicados y porcentajes de cumplimiento

\section{Estándar}

Se cumple

A3. Diseño y métodos. Determinación de la manera en la que se van a encontrar las respuestas a las diferentes preguntas planteadas en la evaluación.

$94,7 \%$

B1. Contexto y antecedentes. Inclusión de factores contextuales que pueden determinar el éxito o el fracaso del programa.

$52,7 \%$

B2. Descripciones y definiciones. Explicitación de los elementos que forman parte del programa y van a ser evaluados.

$94,7 \%$

B3. Clientes. Implicados en la evaluación.

$100 \%$

C1. Procesos. Valor de los medios que emplea la intervención. Se vincula con la evaluación de aquello que ocurre antes de que los resultados finales se produzcan. 
Estándar

Se cumple

C2. Efectos. Evaluación del impacto (efectividad del programa).

$94,7 \%$

D1. Síntesis. Resumen de la importancia relativa de cada dimensión.

$100 \%$

D3. Responsabilidad y justificación. En caso de que la responsabilidad puede ser determinada y sea oportuno hacerlo.

$100 \%$

D4. Informe y apoyos. Esclarecimiento de las conclusiones de manera apropiada, para los grupos, momento y lugar apropiados.

$100 \%$

De la metaevaluación realizada es posible concluir que, si bien las evaluaciones cumplen de manera completa los estándares seleccionados de forma general, existen excepciones. Como puede observarse, solamente en uno de los programas no consta que se cumpla totalmente el estándar A3 (Diseño y métodos), al no detallarse ni el método ni el diseño de la evaluación @conviene recordar que la metaevaluación se basa en la información proporcionada por la literatura, por lo que la determinación de que los aspectos de un estándar no se cumplen puede no adecuarse a la realidad de la evaluación y deberse, en cambio, a la falta de información disponible.

En lo que respecta al estándar B1 (Contexto y antecedentes), en el 52,7\% de las evaluaciones se ha considerado la influencia de los factores contextuales específicos en los que se desarrollan tanto los programas como los procesos de evaluación. El estándar B2 (Descripciones y definiciones) se cumple en el 94,7\% de los casos, describiendo explícitamente las variables consideradas en el proceso, mientras que el 100\% de ellas consideran las perspectivas de los interesados (B3, Clientes).

En lo relativo al estándar C1 (Procesos), tan solo el 42,1\% de las evaluaciones lo cumplen, ya que en el resto se contemplan exclusivamente el diseño del programa o los resultados. En línea con lo anterior, el estándar C2 (Efectos), relacionado con la evaluación de la efectividad del programa, se contempla en el 94,7\% de las evaluaciones.

Finalmente, la totalidad de los procesos de evaluación cumplen los estándares D1 (Síntesis), D2 (Responsabilidad y justificación) y D4 (Informe y apoyos), puesto que en todos ellos se reflejan los resultados de las dimensiones evaluadas, se indican los responsables de las evaluaciones y se establecen de manera clara las conclusiones del proceso.

\section{Discusión y conclusiones}

La evaluación de programas es un campo de estudio extenso y riguroso que requiere de un análisis y aplicación de criterios de evaluación bien seleccionados y definidos. La escasa muestra de trabajos analizados pone de manifiesto la necesidad de realizar evaluaciones rigurosas que contribuyan a constatar la calidad y eficacia de los programas.

El objetivo de este trabajo ha sido analizar los procesos y resultados de evaluación de diferentes programas de educación emocional y motivación dirigidos a estudiantes de Secundaria y/o adolescentes. Entre las evaluaciones identificadas se ha encontrado una gran diversidad de procedimientos de evaluación, así como variadas formas de abordar los resultados ya que, si bien existen algunos aspectos comunes a la mayoría 
de las evaluaciones, las diferencias en el modo de realizar estos procesos son notables, existiendo evaluaciones de tipo cualitativo, cuantitativo o mixto que emplean, por tanto, instrumentos de recogida de información muy variados. En este sentido, aunque la disparidad de las características de las evaluaciones de los programas dificulta realizar generalizaciones, la mayoría de las evaluaciones son sumativas y se centran en la determinación de los resultados del programa, prestando menor atención al diseño y proceso de aplicación. Este aspecto resta calidad a la aplicación de los programas, ya que a través de la evaluación inicial y procesual se garantiza la calidad de su diseño, así como su ajuste a las necesidades iniciales y reajuste a aquellas surgidas durante su aplicación. Además, un elevado número de evaluaciones se han desarrollado de manera interna, de modo que las personas encargadas de su diseño eran, también, los propios evaluadores. Este hecho presenta la ventaja de que los evaluadores conocen profundamente las características del programa y de sus destinatarios, pero cabría cuestionarse si una evaluación externa o mixta aumentaría la objetividad del proceso.

Además, es preciso hacer notar que la difusión de los resultados en medios científicos no se realiza hasta el año 2012, y más concretamente a partir del año 2015, mostrando esta escasa tradición de la literatura académica (Pérez-González, 2008).

Estos resultados están en línea con los hallados por Expósito et al. (2004), quienes analizan los patrones metodológicos de evaluación de 208 estudios publicados entre 1975 y 2000; la metodología empleada fue principalmente cuantitativa, utilizando un modelo evaluativo en la búsqueda de resultados de aplicación. Sin embargo, a diferencia del presente estudio, se emplearon principalmente instrumentos no estandarizados. Otro de los elementos a considerar es la capacidad de generalización de los resultados de los programas, puesto que la aplicación a muestras reducidas o poco representativas no garantiza en absoluto su aplicabilidad; el tamaño reducido de las muestras y el uso generalizado de autoinformes puede no estar mostrando niveles reales de competencia (Torrijos et al., 2018), de modo que es preciso replicar los programas en otras poblaciones para poder asegurar sus resultados de evaluación. En este sentido, las evaluaciones identificadas presentan muestras de diversos tamaños que oscilan entre los 23 y los más de mil sujetos, teniendo estas últimas mayores garantías de calidad.

La metaevaluación permite extraer interesantes conclusiones. Así, en primer lugar, se ha podido determinar que la gran mayoría de evaluaciones cumplen el estándar A3, relativo al Diseño y métodos de la evaluación, y el B2 (Descripciones y definiciones) de la Meta-Evaluation Checklist (Scriven, 2013), determinando los aspectos metodológicos y la descripción de aquello que se evalúa de manera concreta y detallada. Además, todas las evaluaciones cumplen los estándares seleccionados del grupo Conclusiones e implicaciones, contemplando los resultados de cada dimensión, las conclusiones de la evaluación y los responsables de realizarla, así como el estándar B3 (Clientes) del grupo Fundamentos. Se constata asimismo que la mayoría de las evaluaciones se centran en determinar los resultados de los programas, olvidando tanto la evaluación del diseño como de los procesos. Este aspecto merece una especial consideración, ya que no realizar este tipo de evaluaciones implica, por una parte, aplicar programas de los que no se tiene certeza de que sean válidos para alcanzar los objetivos planteados y, por otra, perder la oportunidad de realizar mejoras en el proceso de aplicación del programa. Finalmente, es notable que el estándar Contexto y antecedentes (B1) se tenga en cuenta 
casi en la mitad de las ocasiones. Se trata de un elemento también considerado en los estándares del Joint Committee on Standards for Educational Evaluation (Yarbrough et al., 2010) y con elevada importancia en la evaluación, puesto que contempla las características contextuales a la hora de evaluar y analizar los resultados de evaluación, y pasar por alto este elemento puede suponer que las causas de los resultados se atribuyan a aspectos que no sean sus verdaderos causantes. Es posible que el cumplimiento casi total de determinados indicadores en todos los programas revisados (A3, B2, D1, D3 y D4) se deba, asimismo, a la exigencia de las publicaciones científicas que consideran necesario para su difusión contar con dicha información. Del mismo modo, podría incrementarse la exigencia en el cumplimiento de otros indicadores de evaluación si dichas publicaciones también así lo consideraran, de tal manera que se consiguiera asegurar que los programas aplicados han sido evaluados correctamente, independientemente del modelo de evaluación seleccionado y empleado para ello.

Este trabajo pretende ser un acercamiento a la evaluación de programas de educación emocional y motivación que, para confirmar estos resultados, requerirá ampliar la muestra de evaluaciones seleccionadas a través de revisiones sistemáticas que permitan analizar las evaluaciones de los programas en profundidad y aplicar los estándares de metaevaluación de manera completa.

Dada la abundante publicación de programas de intervención, será necesario seguir desarrollando una cultura de investigación evaluativa entre los diferentes profesionales de la educación (Manzano, 2000), poniendo el énfasis en la formación correcta de futuros evaluadores (LaVelle, 2019), de tal manera que puedan conocer las bases teóricas de los modelos de evaluación que emplean para evaluar con confianza en función de las necesidades y de la complejidad de los programas (Frye y Hammer, 2012). En este sentido, sería necesario que no solo tuvieran conocimientos de los modelos y elementos de la evaluación sino, también, de los diferentes procedimientos de metaevaluación existentes para garantizar su calidad y rigor. Para ello, se les debería proporcionar formación tanto teórica como práctica en contextos educativos reales. Además, sería de interés para los investigadores poner el foco en la evaluación de programas y no tanto en la creación o diseño de nuevos instrumentos de intervención que, en muchas ocasiones, se quedan sin posteriores aplicaciones. Contribuir a la comunidad científica no siempre supone la elaboración de un nuevo instrumento o programa; una adecuada contribución es alimentar la producción obteniendo y validando herramientas de valor para su aplicación real en el contexto educativo.

Desde un punto de vista más aplicado y práctico, y ante la importante tarea de selección y aplicación de programas de intervención que tienen por delante orientadores, pedagogos y educadores en general, disponer de estudios sólidos que acompañen los resultados de aplicación de los programas y que pongan su foco en la evaluación de los mismos, podrá ser una herramienta útil para la toma de decisiones. 


\section{Referencias}

Álvarez Rojo, V., García, E. Gil, J., Martínez, P., Romero, S., y Rodríguez, J. (2002). Diseño y evaluación de programas. Madrid: EOS

Álvarez Rojo, V., y Hernández Fernández, J. (1998). El modelo de intervención por programas. Aportaciones para una revisión. Revista de Investigación Educativa, 16(2), 79-123.

Anaya D. (2014). Bases del aprendizaje y educación. Madrid: UNED

Barati, M., Joo, S. B., y Aria, A. M. (2018). Studying the impact of metacognitive beliefs and self-regulatory strategies on students' educational motivation. Iranian Journal of Social Sciences and Humanities Research, 6(1).

Burunat, E. (2004). El desarrollo del sustrato neurobiológico de la motivación y emoción en la adolescencia: ¿un nuevo período crítico? Infancia y aprendizaje, 27(1), 87-104.

De Caso, A. M. (2014). Pautas para el estudio de la motivación académica. International Journal of Developmental and Educational Psychology. Revista INFAD de Psicología., 6(1), 213-220, doi: 10.17060/ijodaep.2014.n1.v6.736

Expósito, J., Olmedo, E., y Cano, A. F. (2004). Patrones metodológicos en la investigación española sobre evaluación de programas educativos. RELIEVE, 10(2), 185-209, doi: 10.7203/relieve.10.2.4319

Frye, A. W., y Hemmer, P. A. (2012). Program evaluation models and related theories: AMEE guide no. 67. Medical teacher, 34(5), e288-e299, doi: 10.3109/0142159X.2012.668637

García, F. (2008). Motivar para el aprendizaje desde la acción orientadora. Ministerio de Educación y Ciencia: Madrid.

Järvelä, S. (2001). Shifting research on motivation and cognition to an integrated approach on learning and motivation in context. En Volet, S., y Järvelä, S. (Eds.), Motivation in learning contexts: Theoretical advances and methodological implications. London: Pergamon.

Kirkpatrick, D.L. (1994). Evaluating Training Programs: The Four Levels. San Francisco, CA: Berrett-Koehler.

Lange, J., Dalege, J., Borsboom, D., van Kleef, G. A., y Fischer, A. H. (2020). Toward an Integrative Psychometric Model of Emotions. Perspectives on Psychological Science, 15(2), 444-468.

LaVelle, J.M. (2019). Educating Evaluators 1976-2017: An Expanded Analysis of University-Based Evaluation Education Programs. American Journal of Evaluation 20(10), 1-16, doi: 10.1177/1098214019860914

López, J. G. (2006). Aportaciones de la teoría de las atribuciones causales a la comprensión de la motivación para el rendimiento escolar. Ensayos: Revista de la Facultad de Educación de Albacete (21), 217-232.

Manzano, N. (2000). Indicadores para evaluar programas de orientación educativa. Revista de Orientación Educativa y Psicopedagógica, (19), 51-75.

Martínez Mediano, C. (2017). Evaluación de programas. Madrid: UNED.

Méndez Pineda, J. M. (2018). Evaluación externa del programa escuelas de tiempo completo, 2016. Recuperado de: http://www.seslp.gob.mx/pdf/informe_evaluacionExterna.pdf

Mirete Ruiz, A. B., Soro Bernal, M., y Maquilón Sánchez, J. J. (2015). El fracaso escolar y los enfoques de aprendizaje: medidas para la inclusión educativa. Revista Electrónica Interuniversitaria de formación del profesorado, 18(3), 183-196. doi: 10.6018/reifop.18.3.239021 
OMS (2020). Adolescence health. Recuperado de: https://www.who.int/health-topics/ adolescent-health\#tab=tab_1

Pérez Juste, R. (1995) Metodología para la evaluación de programas educativos. En A. Medina y L.M. Villar. Evaluación de Programas Educativos, Centros y Profesores. Madrid: Universitas

Pérez Juste (2000). La evaluación de programas educativos: conceptos básicos, planteamientos generales y problemática. Revista de investigación educativa, 18(2), 261-287.

Pérez Juste (2006). Evaluación de programas educativos. Madrid: La muralla.

Pérez-González, J. C. (2008). Propuesta para la evaluación de programas de educación socioemocional. Electronic Journal of Research in Education Psychology, 6(15), 523-546.

Pulido Acosta, F., y Herrera Clavero, F. (2017). La influencia de las emociones sobre el rendimiento académico. Ciencias Psicológicas, 11(1), $29-39$.

Rinaudo, M. C., Chiecher, A., y Donolo, D. (2003). Motivación y uso de estrategias en alumnos universitarios. Su evaluación a partir del Motivated Strategies Learning Questionnaire. Anales de psicología, 19(1), 107-119.

Sánchez Calleja, L., García Jiménez, E., y Rodríguez Gómez, G. (2016). Evaluación del diseño del programa AedEM de Educación Emocional para Educación Secundaria. Relieve: Revista Electrónica de Investigación y Evaluación Educativa, 22 (2), 1-25, doi: 10.7203/relieve.22.2.9422

Samson, B., y Solabarrieta, J. (2018). Improving evaluation of students: a meta-evaluation study on cce from the perspective of students evaluation standards. MIER Journal of Educational Studies, Trends and Practices, 8(2).

Sawyer, S. M., Azzopardi, P. S., Wickremarathne, D., y Patton, G. C. (2018). The age of adolescence. The Lancet Child \& Adolescent Health, 2(3), 223-228.

Scriven, M. (1967). The methodology of evaluation. En Tyler, R. W., Gagné, R., W., y Scriven, M. (Eds.). Perspectives of curriculum evaluation. Chicago. Rand McNally.

Scriven, M. (2013). Key evaluation checklist (KEC). Recuperadon de: www.michaelscriven. info/images/KEC_3.22.2013.pdf

Stake, R.E. (1975) To Evaluate an Arts Program. En R.E. Stake (Ed.), Evaluating the Arts in Education: A Responsive Approach. Columbus, $\mathrm{OH}$ : Merrill.

Stufflebeam, D. L. (1971). The relevance of the CIPP evaluation model for educational accountability. En Annual meeting of the American Association of School Administrators.

Stufflebeam, D. L. (1987). Professional standards for assuring the quality of educational program and personnel evaluations. International Journal of Educational Research, 11(1), 125-143.

Tejedor, F. J. (2000). El diseño y los diseños en la evaluación de programas. Revista de investigación educativa, 18(2), 319-339.

Torrijos, P., Torrecilla, E. M., y Rodríguez, M. J. (2018). Experimental evaluation of emotional development programmes for teachers in Secondary Education. anales de psicología, 34(1), 68-76, doi: 10.6018/analesps.34.1.273451

Yarbrough, D.B., Shula, L.M., Hopson, R.K., \& Caruthers, F.A. (2010). The Program Evaluation Standards: A guide for evaluators and evaluation users (3rd. ed). Thousand Oaks, CA: Corwin Press. 


\section{Apéndice}

Andersen, F. Ø., Nissen, P., y Poulsen, L. (2016). Inclusion of Marginalized Boys: A Survey of a Summer School Using Positive Psychology Interventions. Journal of Educational Issues, 2(1), 231247.

\begin{tabular}{|c|c|}
\hline Nombre & The Boys Academy \\
\hline País & Dinamarca \\
\hline Metodología y diseño & $\begin{array}{l}\text { Metodología mixta, diseño pre-experimental de un solo grupo con } \\
\text { pre test-mid test-post test }\end{array}$ \\
\hline Muestra & 100. Edad: $14-16$ años \\
\hline Instrumentos & $\begin{array}{l}\text { Test de rendimiento, entrevistas, escala de aprendizaje (Larning } \\
\text { Rating Scale) }\end{array}$ \\
\hline Evaluador & Interna \\
\hline Función & Sumativa \\
\hline Perspectiva & Ex post \\
\hline Elementos & Resultados \\
\hline Estándares & $\mathrm{A} 3, \mathrm{~B} 1, \mathrm{~B} 2, \mathrm{~B} 3, \mathrm{C} 2, \mathrm{D} 1, \mathrm{D} 3, \mathrm{D} 4$ \\
\hline \multicolumn{2}{|c|}{$\begin{array}{c}\text { Armada, J. M. (2017). La Expresión Corporal como herramienta para el desarrollo de habilidades } \\
\text { socioafectivas en el alumnado de educación secundaria obligatoria. Tesis doctoral. Universidad de } \\
\text { Córdoba, Córdoba. }\end{array}$} \\
\hline Nombre & Programa de Expresión Corporal Anaya \\
\hline País & España \\
\hline Metodología y diseño & $\begin{array}{l}\text { Metodología mixta, diseño pre-experimental de un solo grupo con } \\
\text { pre-test y post-test }\end{array}$ \\
\hline Muestra & 23. Edad: cuarto de secundaria \\
\hline Instrumentos & $\begin{array}{l}\text { Escala de Medición de Habilidades Socioafectivas, grupo de } \\
\text { discusión, cuestionario de satisfacción }\end{array}$ \\
\hline Evaluador & Interna \\
\hline Función & Sumativa \\
\hline Perspectiva & Ex post \\
\hline Elementos & Implementación y resultados \\
\hline Estándares & $\mathrm{A} 3, \mathrm{~B} 1, \mathrm{~B} 2, \mathrm{~B} 3, \mathrm{C} 1, \mathrm{C} 2, \mathrm{D} 1, \mathrm{D} 3, \mathrm{D} 4$ \\
\hline
\end{tabular}


Cejudo, J. (2015). Diseño, desarrollo y evaluación del programa "Dulcinea" de educación emocional en un centro de educación secundaria desde la integración de modelos de inteligencia emocional. Tesis doctoral. Madrid: Universidad Nacional de Educación a distancia.

\begin{tabular}{|c|c|}
\hline Nombre & Programa Dulcinea \\
\hline País & España \\
\hline Metodología y diseño & $\begin{array}{l}\text { Metodología cuantitativa, diseño cuasiexperimental: pre test-post } \\
\text { test con grupos de control no equivalentes }\end{array}$ \\
\hline Muestra & 507. Edad: no consta \\
\hline Instrumentos & $\begin{array}{l}\text { Mayer Salovey Caruso Emotional Intelligence Test, Situational } \\
\text { Test of Emotional Understanding, Trait Emotional Intelligence } \\
\text { Questionnaire Adolescents Short Form, Mental Health-5, } \\
\text { Cuestionario de Sensibilidad al Castigo y Sensibilidad a la } \\
\text { Recompensa para jóvenes, Primary Mental Abilities, Cuestionario } \\
\text { factorial de personalidad v.5, Escalas de Estrategias Motivacionales } \\
\text { del Aprendizaje, Sistema de evaluación de la conducta de niños } \\
\text { y adolescentes, test de conocimiento explícito emocional para } \\
\text { adolescentes (adaptaciones al castellano), calificaciones, faltas de } \\
\text { asistencia, partes de amonestación, cuestionario sociométrico }\end{array}$ \\
\hline Evaluador & Interna \\
\hline
\end{tabular}

\begin{tabular}{ll}
\hline Función & Formativa y sumativa \\
\hline Perspectiva & Ex ante y ex post \\
\hline Elementos & Diseño, implementación y resultados \\
\hline Estándares & A3, B1, B2, B3, C1, C2, D1, D3, D4 \\
\hline $\begin{array}{l}\text { Cleary, T. J., Platten, P., y Nelson, A. (2008). Effectiveness of the self-regulation empowerment } \\
\text { program with urban high school students. Journal of advanced academics, 20(1), 70-107. }\end{array}$ \\
\hline Nombre & Self-regulation empowerment program \\
\hline País & Estados Unidos \\
\hline Metodología y diseño & $\begin{array}{l}\text { Metodología mixta, diseño cuasiexperimental: pre test-post test con } \\
\text { grupo de control no equivalente }\end{array}$ \\
\hline Muestra & $\begin{array}{l}\text { 15. Edad: no consta } \\
\text { Instrumentos }\end{array}$ \\
$\begin{array}{l}\text { Wisconsin Knowledge and Concepts Examination, Self-Regulaion } \\
\text { Strategu Interventory-Self.Report, Rating Student Self-Regulated } \\
\text { Learning Outcomes, Self-Efficacy for Self-Regulated Learning, Self- } \\
\text { Efficacy for Outcomes, Task Interest Inventory, notas de campo, } \\
\text { procedimientos microanalíticos de evaluación, escalas tipo Likert, } \\
\text { calificaciones en los exámenes }\end{array}$ \\
\hline
\end{tabular}




\begin{tabular}{|c|c|}
\hline Evaluador & Interna y externa \\
\hline Función & Sumativa \\
\hline Perspectiva & Ex post \\
\hline Elementos & Resultados \\
\hline Estándares & $\mathrm{A} 3, \mathrm{~B} 1, \mathrm{~B} 2, \mathrm{~B} 3, \mathrm{C} 2, \mathrm{D} 1, \mathrm{D} 3, \mathrm{D} 4$ \\
\hline \multicolumn{2}{|c|}{$\begin{array}{l}\text { Deutsch, N. L., Reitz-Krueger, C. L., Henneberger, A. K., Futch Ehrlich, V. A., y Lawrence, E. } \\
\text { C. (2017). "It Gave Me Ways to Solve Problems and Ways to Talk to People" Outcomes from a } \\
\text { Combined Group and One-on-One Mentoring Program for Early Adolescent Girls. Journal of } \\
\text { Adolescent Research, 32(3), 291-322. }\end{array}$} \\
\hline Nombre & Young Women Leaders Program \\
\hline País & Estados Unidos \\
\hline Metodología y diseño & $\begin{array}{l}\text { Metodología cualitativa, diseño pre-experimental de un solo grupo } \\
\text { con post-test }\end{array}$ \\
\hline Muestra & Número de estudiantes: 113. Edad: séptimo curso de secundaria \\
\hline Instrumentos & Entrevistas semiestructuradas \\
\hline Evaluador & Externa \\
\hline Función & Sumativa \\
\hline Perspectiva & Ex post \\
\hline Elementos & Resultados \\
\hline Estándares & $\mathrm{A} 3, \mathrm{~B} 1, \mathrm{~B} 2, \mathrm{~B} 3, \mathrm{C} 2, \mathrm{D} 1, \mathrm{D} 3, \mathrm{D} 4$ \\
\hline \multicolumn{2}{|c|}{$\begin{array}{c}\text { de Fátima, M., de Jesus, S. N., Azevedo, I., Araújo, A. M., y Viseu, J. (2015). Intervention } \\
\text { Program on Adolescent's Creativity Representations and Academic Motivation. Paidéia (Ribeirão } \\
\text { Preto), 25(62), 289-298. }\end{array}$} \\
\hline Nombre & Future Problem Solving \\
\hline País & Portugal \\
\hline Metodología y diseño & $\begin{array}{l}\text { Metodología cuantitativa, diseño cuasiexperimental: pre test-post } \\
\text { test con grupo de control no equivalente }\end{array}$ \\
\hline Muestra & 155. Edad: $12-17$ años \\
\hline Instrumentos & $\begin{array}{l}\text { School and Creativity Scale-Students' Perceptions, Scale of } \\
\text { Motivation for Academic Learning }\end{array}$ \\
\hline Evaluador & Externa \\
\hline Función & Sumativa \\
\hline
\end{tabular}




\begin{tabular}{|c|c|}
\hline Perspectiva & Ex post \\
\hline Elementos & Resultados \\
\hline Estándares & $\mathrm{A} 3, \mathrm{~B} 2, \mathrm{~B} 3, \mathrm{C} 2, \mathrm{D} 1, \mathrm{D} 3, \mathrm{D} 4$ \\
\hline \multicolumn{2}{|c|}{$\begin{array}{l}\text { Godás-Otero, A., Lorenzo-Moledo, M., y Priegue-Caamaño, D. (2015). Efectos del programa } \\
\text { ECO-FA-SE sobre la autoestima académica y la percepción de implicación familiar. Revista de } \\
\text { Estudios e Investigación en Psicología y Educación, (5), 145-148. }\end{array}$} \\
\hline Nombre & Programa ECO-FA-SE \\
\hline País & España \\
\hline Metodología y diseño & $\begin{array}{l}\text { Metodología cuantitativa, diseño cuasiexperimental: pre test-post } \\
\text { test con grupo de control no equivalente }\end{array}$ \\
\hline Muestra & Tres institutos. Edad: $1^{\circ}$ y $2^{\circ}$ de secundaria \\
\hline Instrumentos & $\begin{array}{l}\text { Calificaciones, escala de autoestima académica, escala de percepción } \\
\text { de implicación familiar }\end{array}$ \\
\hline Evaluador & Interna \\
\hline Función & Sumativa \\
\hline Perspectiva & Ex post \\
\hline Elementos & Resultados \\
\hline Estándares & $\mathrm{A} 3, \mathrm{~B} 2, \mathrm{~B} 3, \mathrm{C} 2, \mathrm{D} 1, \mathrm{D} 3, \mathrm{D} 4$ \\
\hline \multicolumn{2}{|c|}{$\begin{array}{l}\text { Iriarte Redín, C. y Benavides, M. (2011). Evaluación del programa para superar la ansiedad } \\
\text { hacia las matemáticas PAM. International Journal of Developmental and Educational Psychology: } \\
\text { INFAD. Revista de Psicología, 2(1), 65-73. }\end{array}$} \\
\hline Nombre & Programa para superar la ansiedad hacia las matemáticas PAM \\
\hline País & España \\
\hline Metodología y diseño & Metodología cuantitativa, diseño no experimental: descriptivo \\
\hline Muestra & 51. Edad: 6-23 años \\
\hline Instrumentos & No consta \\
\hline Evaluador & Interna y externa \\
\hline Función & Sumativa \\
\hline Perspectiva & Ex post \\
\hline Elementos & Implementación y resultados \\
\hline
\end{tabular}


López de la Osa, X. R., y Gutiérrez-Sánchez, Á. (2015). Implementación de un programa de Gimnasia Acrobática en Educación Secundaria para la mejora del autoconcepto. RETOS. Nuevas Tendencias en Educación Física, Deporte y Recreación, (27), 114-117.

\begin{tabular}{|c|c|}
\hline Nombre & Programa de Gimnasia Acrobática \\
\hline País & España \\
\hline Metodología y diseño & $\begin{array}{l}\text { Metodología cuantitativa, diseño pre-experimental de un solo grupo } \\
\text { con pre test-post test }\end{array}$ \\
\hline Muestra & 36. Edad: $14-17$ años \\
\hline Instrumentos & Cuestionario Autoconcepto Forma 5, Registro anecdótico \\
\hline Evaluador & Interna \\
\hline Función & Sumativa \\
\hline Perspectiva & Ex post \\
\hline Elementos & Resultados \\
\hline Estándares & $\mathrm{A} 3, \mathrm{~B} 1, \mathrm{~B} 2, \mathrm{~B} 3, \mathrm{C} 1, \mathrm{C} 2, \mathrm{D} 1, \mathrm{D} 3, \mathrm{D} 4$ \\
\hline \multicolumn{2}{|c|}{$\begin{array}{l}\text { Martinsone, B. (2016). Social emotional learning: Implementation of sustainability-oriented } \\
\text { program in Latvia. Journal of Teacher Education for Sustainability, 18(1), 57-68. }\end{array}$} \\
\hline Nombre & Social Emotional Learning Program \\
\hline País & Letonia \\
\hline Metodología y diseño & Metodología mixta \\
\hline Muestra & 12699. Edad: no consta \\
\hline Instrumentos & No consta \\
\hline Evaluador & Interna \\
\hline Función & Sumativa \\
\hline Perspectiva & Ex ante y ex post \\
\hline Elementos & Resultados \\
\hline Estándares & $\mathrm{B} 2, \mathrm{~B} 3, \mathrm{C} 2, \mathrm{D} 1, \mathrm{D} 3, \mathrm{D} 4$ \\
\hline \multicolumn{2}{|c|}{$\begin{array}{l}\text { Miloseva, L. (2013). A Cognitive Behavioral Depression Prevention Program for Early } \\
\text { Adolescents. Journal on Educational Psychology, 6(3), 32-40. }\end{array}$} \\
\hline Nombre & Cognitive Behavioral Depression Prevention Program \\
\hline País & Macedonia \\
\hline
\end{tabular}




\begin{tabular}{|c|c|}
\hline Metodología y diseño & $\begin{array}{l}\text { Metodología cuantitativa, diseño pre-experimental de un solo grupo } \\
\text { con pre test-post test }\end{array}$ \\
\hline Muestra & 128. Edad: 14 años \\
\hline Instrumentos & Children 's Attribution Style Questionnaire \\
\hline Evaluador & Interna \\
\hline Función & Sumativa \\
\hline Perspectiva & Ex post \\
\hline Elementos & Resultados \\
\hline Estándares & $\mathrm{A} 3, \mathrm{~B} 2, \mathrm{~B} 3, \mathrm{C} 2, \mathrm{D} 1, \mathrm{D} 3, \mathrm{D} 4$ \\
\hline \multicolumn{2}{|c|}{$\begin{array}{l}\text { Rodríguez-Ledo, C., Orejudo Hernández, S., Celma Pastor, L., y Cardoso Moreno, M. J. (2018). } \\
\text { Mejora de las competencias socioemocionales en el aula, con jóvenes de educación secundaria, } \\
\text { mediante el programa SEA. Electronic Journal of Research in Educational Psychology, 16(3), 681- } \\
701 .\end{array}$} \\
\hline Nombre & Programa SEA \\
\hline País & España \\
\hline Metodología y diseño & $\begin{array}{l}\text { Metodología cuantitativa, diseño cuasiexperimental: pre test-post } \\
\text { test con grupo de control no equivalente. }\end{array}$ \\
\hline Muestra & 156. Edad: $11-14$ años \\
\hline Instrumentos & $\begin{array}{l}\text { Cuestionario de Desarrollo Emocional para Secundaria, Emotional } \\
\text { Quotient Inventory: Youth Version EQi- YV, Batería de socialización } \\
\text { BAS3, Index of Empathy for Children and Adolescents }\end{array}$ \\
\hline Evaluador & Interna y externa \\
\hline Función & Sumativa \\
\hline Perspectiva & Ex post \\
\hline Elementos & Resultados \\
\hline Estándares & A3, B2, B3, C2, D1, D3, D4 \\
\hline \multicolumn{2}{|c|}{$\begin{array}{l}\text { Rueda Carcelén, P. M., Cabello, E., Filella Guiu, G., y Ros Morente, A. (2017). Los programas } \\
\text { de educación emocional Happy 8-12 y Happy 12-16 para la resolución asertiva de conflictos en } \\
\text { niños y adolescentes. Innovación educativa, 27, 75-79. }\end{array}$} \\
\hline Nombre & Happy 12-16 \\
\hline País & España \\
\hline Metodología y diseño & $\begin{array}{l}\text { Metodología mixta, diseño experimental: pre test-post test con } \\
\text { grupo de control equivalente }\end{array}$ \\
\hline
\end{tabular}




\begin{tabular}{|c|c|}
\hline Muestra & 903. Edad: primero y segundo de secundaria \\
\hline Instrumentos & Cuestionarios estructurados, entrevistas estructuradas \\
\hline Evaluador & Interna \\
\hline Función & Sumativa \\
\hline Perspectiva & Ex post \\
\hline Elementos & Resultados \\
\hline Estándares & $\mathrm{A} 3, \mathrm{~B} 2, \mathrm{~B} 3, \mathrm{C} 2, \mathrm{D} 1, \mathrm{D} 3, \mathrm{D} 4$ \\
\hline \multicolumn{2}{|c|}{$\begin{array}{l}\text { Ruiz-Aranda, D., Salguero, J. M., Cabello, R., Palomera, R., y Berrocal, P. F. (2012). Can an } \\
\text { emotional intelligence program improve adolescents' psychosocial adjustment? results from the } \\
\text { INTEMO project. Social Behavior and Personality: an international journal, 40(8), 1373-1379. }\end{array}$} \\
\hline Nombre & Proyecto Intemo \\
\hline País & España \\
\hline Metodología y diseño & $\begin{array}{l}\text { Metodología cuantitativa, diseño cuasiexperimental: pre test-post } \\
\text { test con grupo de control no equivalente }\end{array}$ \\
\hline Muestra & 147. Edad: $13-16$ años \\
\hline Instrumentos & Behavior Assessment System for Children and Adolescents \\
\hline Evaluador & Interno y externo \\
\hline Función & Sumativa \\
\hline Perspectiva & Ex post \\
\hline Elementos & Resultados \\
\hline Estándares & A3, B2, B3, C2, D1, D3, D4 \\
\hline \multicolumn{2}{|c|}{$\begin{array}{c}\text { Sánchez-Calleja, L., García-Jiménez, E., y Rodríguez-Gómez, G. (2016). Evaluación del diseño } \\
\text { del programa AedEM de Educación Emocional para Educación Secundaria. Relieve: Revista } \\
\text { Electrónica de Investigación y Evaluación Educativa, 22(2), 1-25. }\end{array}$} \\
\hline Nombre & Programa EADEM \\
\hline País & España \\
\hline Metodología y diseño & Metodología cuantitativa, diseño no experimental \\
\hline Muestra & Validación por jueces \\
\hline Instrumentos & Escala tipo Likert \\
\hline Evaluador & Interna y externa \\
\hline
\end{tabular}




\begin{tabular}{|c|c|}
\hline Función & Formativa \\
\hline Perspectiva & Ex ante \\
\hline Elementos & Diseño \\
\hline Estándares & $\mathrm{A} 3, \mathrm{~B} 1, \mathrm{~B} 2, \mathrm{~B} 3, \mathrm{C} 2, \mathrm{D} 1, \mathrm{D} 3, \mathrm{D} 4$ \\
\hline \multicolumn{2}{|c|}{$\begin{array}{l}\text { Sibieta, L., Greaves, E., y Sianesi, B. (2014). Increasing Pupil Motivation: Evaluation Report and } \\
\text { Executive Summary. London: Education Endowment Foundation. }\end{array}$} \\
\hline Nombre & Increasing Pupil Motivation \\
\hline País & Reino Unido \\
\hline Metodología y diseño & $\begin{array}{l}\text { Metodología mixta, diseño cuasiexperimental: pre test-post test con } \\
\text { grupo de control no equivalente }\end{array}$ \\
\hline Muestra & 63 escuelas. Edad: Year 11 \\
\hline Instrumentos & Exámenes de GCSE, entrevistas semiestructuradas \\
\hline Evaluador & Interna \\
\hline Función & Formativa y sumativa \\
\hline Perspectiva & Ex post \\
\hline Elementos & Implementación y resultados \\
\hline Estándares & $\mathrm{A} 3, \mathrm{~B} 1, \mathrm{~B} 2, \mathrm{~B} 3, \mathrm{C} 1, \mathrm{C} 2, \mathrm{D} 1, \mathrm{D} 3, \mathrm{D} 4$ \\
\hline \multicolumn{2}{|c|}{$\begin{array}{l}\text { Sorensen, N., Oyserman, D., Eisner, R., Yoder, N., y Horowitz, E. (2018). Pathways to } \\
\text { Success: Developing and Testing a Scalable Identity-Based Motivation Intervention in the } \\
\text { Classroom. Society for Research on Educational Effectiveness.Spring } 2018 \text { Conference Abstract. }\end{array}$} \\
\hline Nombre & Pathways to Success \\
\hline País & Estados Unidos \\
\hline Metodología y diseño & $\begin{array}{l}\text { Metodología cuantitativa, diseño cuasiexperimental: pre test-post } \\
\text { test con grupo de control no equivalente }\end{array}$ \\
\hline Muestra & 380. Edad: octavo curso \\
\hline Instrumentos & Videos de las clases \\
\hline Evaluador & Externa \\
\hline Función & Sumativa \\
\hline Perspectiva & Ex post \\
\hline Elementos & Implementación y resultados \\
\hline
\end{tabular}




\begin{tabular}{|c|c|}
\hline Estándares & $\mathrm{A} 3, \mathrm{~B} 1, \mathrm{~B} 2, \mathrm{~B} 3, \mathrm{C} 1, \mathrm{C} 2, \mathrm{D} 1, \mathrm{D} 3, \mathrm{D} 4$ \\
\hline \multicolumn{2}{|c|}{$\begin{array}{l}\text { Wilkens, C. L., y Wilmore, E. (2015). Does Implementing an Emotional Intelligence Program } \\
\text { Guarantee Student Achievement? Education Leadership Review of Doctoral Research, 2(1), 34-46. }\end{array}$} \\
\hline Nombre & The Leader in Me \\
\hline País & Estados Unidos \\
\hline Metodología y diseño & $\begin{array}{l}\text { Metodología cuantitativa, diseño cuasiexperimental con grupo de } \\
\text { control no equivalente }\end{array}$ \\
\hline Muestra & No consta. Edad: quinto curso \\
\hline Instrumentos & Informes de diferentes fuentes y resultados académicos \\
\hline Evaluador & Externa \\
\hline Función & Sumativa \\
\hline Perspectiva & Ex ante y ex post \\
\hline Elementos & Implementación y resultados \\
\hline Estándares & $\mathrm{A} 3, \mathrm{~B} 2, \mathrm{~B} 3, \mathrm{C} 1, \mathrm{C} 2, \mathrm{D} 1, \mathrm{D} 3, \mathrm{D} 4$ \\
\hline \multicolumn{2}{|c|}{$\begin{array}{l}\text { Willard, J. A., Bayes, B., y Martinez, J. (2015). Gateway to College: Lessons from Implementing a } \\
\text { Rigorous Academic Program for At-Risk Young People. New York: MDRC. }\end{array}$} \\
\hline Nombre & Gateway to College \\
\hline País & Estados Unidos \\
\hline Metodología y diseño & $\begin{array}{l}\text { Metodología mixta, diseño experimental: pre test-mid test-post test } \\
\text { con grupo de control equivalente }\end{array}$ \\
\hline Muestra & No consta. Edad: 16-20 años \\
\hline Instrumentos & $\begin{array}{l}\text { Entrevistas, grupos de discusión, observación, formularios, } \\
\text { cuestionarios, resultados académicos }\end{array}$ \\
\hline Evaluador & Interna \\
\hline Función & Formativa y Sumativa \\
\hline Perspectiva & Ex post \\
\hline Elementos & Implementación \\
\hline Estándares & A3, B1, B3, C1, D1, D3, D4 \\
\hline
\end{tabular}

